\title{
Confocal Holography
}

\author{
Rodney A. Herring and Denis Laurin
}

Canadian Space Agency, St.-Hubert, QC, Canada, J3Y 8 Y9

A new type of microscope, referred to as a Confocal Holography $(\mathrm{CH})$ microscope, has been constructed at the Canadian Space Agency and is now being tested for use in experiments involving the non-intrusive measurement of temperatures and compositions in objects in order to better diagnose mass and heat transfer mechanisms. This microscope is being tested for its ability to generate three-dimensional amplitude and phase images from holograms taken from points on the surfaces, as well as, from points inside transparent objects. Combining the design concepts of confocal scanning Laser microscopes and holography microscopes created the $\mathrm{CH}$ microscope. The $\mathrm{CH}$ microscope may enable for the first time the measurement of both the phase and amplitude of a beam inside an object. The complete amplitude and phase image of the object consists of all the measured amplitudes and phases of the three-dimensional points, combined in proper registry. From the phase information obtained, the refractive index of the object can be determined, which can be used to obtain the object's absolute state, such as its absolute temperature and absolute composition, necessary information for high-level science experiments, studying heat and mass transport.

The combination of a confocal microscope and a holography microscope, as originally proposed by Herring, has been used to create the confocal holography microscope [1]. The Confocal Scanning Laser Microscope uses a Laser beam passing through an object to create three-dimensional amplitude images of objects (up to $0.2 \mu \mathrm{m}$ lateral resolution and $0.4 \mu \mathrm{m}$ in depth), by means of a pinhole $(\mathrm{PH})$ aperture, placed confocal with a point on a focal plane in an object (Figure 1) [2]. This type of microscope, developed during the 1980s and 1990s, has become very useful in the biological, medical and materials sciences, as it can more clearly see three-dimensional objects than by the use of traditional optical microscopes. Standard holography microscopes have been used to measure both the phase and the amplitude of objects, however, these microscopes are only able to measure the beam's amplitude and phase arising from the object's surfaces or from the integrated through-thickness of the object, i.e., the object as a whole, and not at points within the object.[3, 4] A confocal holography microscope (Figure 2) is being used to measure both the phase and amplitude of a beam at points inside reference objects comprising of a flame, and a fluid cell having a temperature gradient. Certainty of this measurement is not guaranteed, as the method creates a scientific anomaly given that the Laser beams are detected after they have passed through the entire object. Information transfer theory for weak phase objects is being considered for the amplitude and phase information. [5]

\section{References}

[1] R. A. Herring, Optik 105 (1997) 65-68.

[2] A.E. Dixon et al., Journal of Microscopy 178 (1995) 161-266.

[3] Introduction to Holography, Editors E. Voelk1, L. F. Allard, D. C. Joy, (Kluwer Academic/Plenum Publishers, New York 1999).

[4] A. Tonomura, Electron Holography, Editor K. Shimoda, Springer Series in Optical Sciences V 70 (1993).

[5] H. Lichte, Advances in Optical and Electron Microscopy (Academic Press) V 12 (1991) 25-91. 


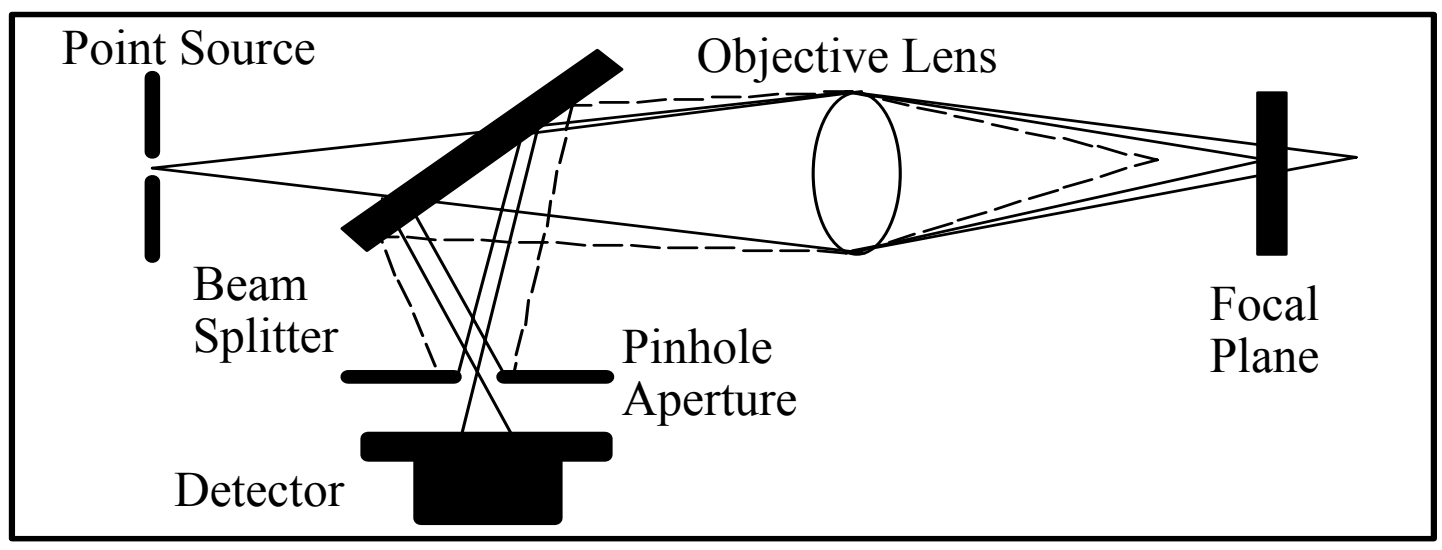

FIG. 1. A Confocal Microscope showing its general principles, where only the beam focussed to a point at the focal plane is confocal to the Pinhole Aperture and thus allowed to pass though to the detector.

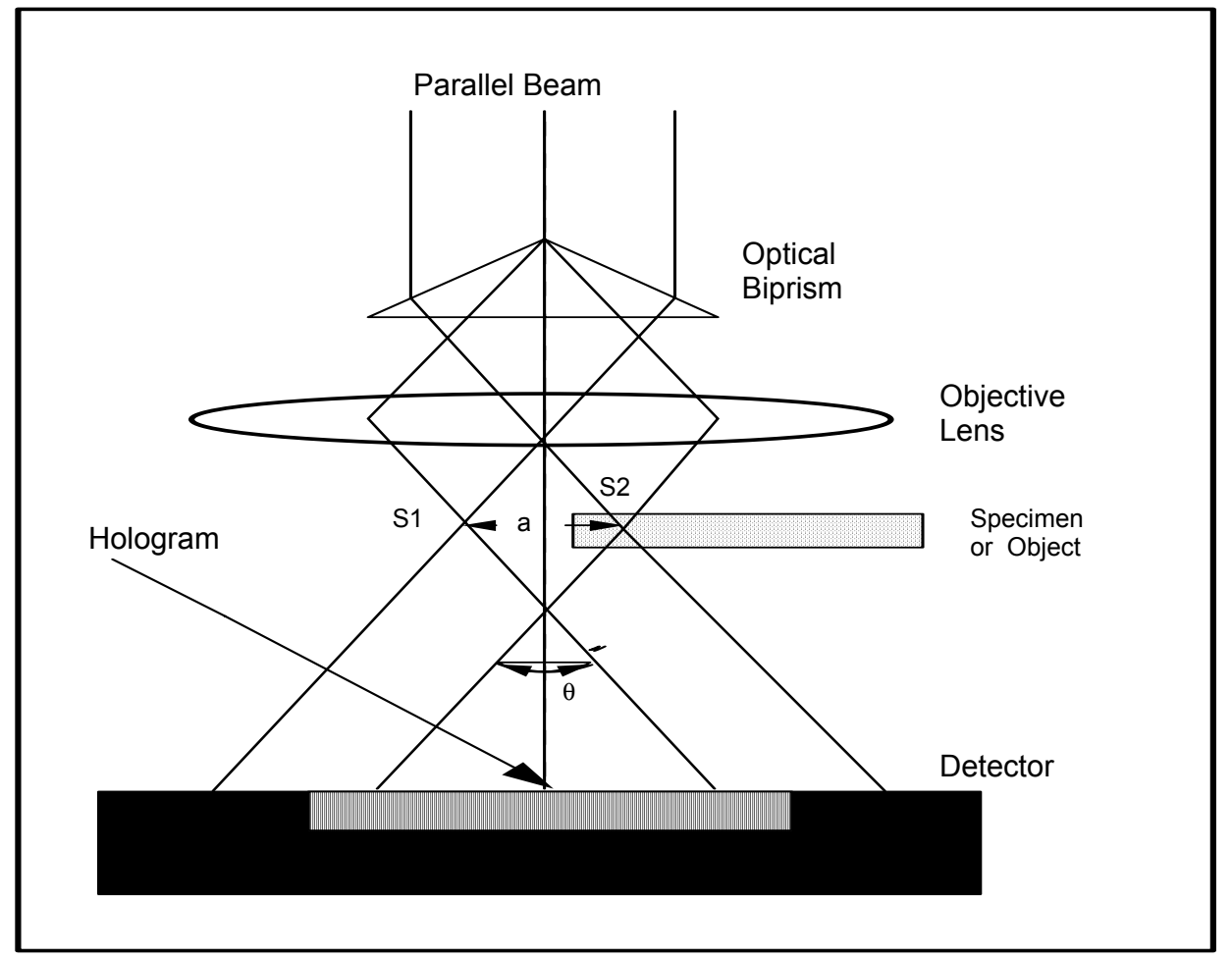

FIG. 2. A parallel beam is split into two by a biprism, one going to the right and the other going to the left, until they are redirected and focussed by the objective lens into two probes, one passing through the specimen (object wave) and the other passing to the side of the specimen (reference wave). The object wave and reference wave then interfere at the detector to form a hologram. 